\title{
Prediction of clinical height gain from surgical posterior correction of idiopathic scoliosis
}

\author{
Tristan Langlais, MD, MSc, ${ }^{1,2,4}$ Stephane Verdun, PhD, ${ }^{3}$ Roxane Compagnon, MD, MSc, ${ }^{4}$ \\ Catalin Ursu, MD, ${ }^{1}$ Claudio Vergari, PhD, ${ }^{2}$ Hugo Barret, MD, MSc, ${ }^{5}$ and Christian Morin, MD1 \\ ${ }^{1}$ Pediatric Orthopaedics Department, Institut Calot, Berck-sur-Mer; ${ }^{2}$ Institut de Biomécanique Humaine Georges Charpak, \\ Arts et Metiers ParisTech, Laboratoire de Biomécanique, Paris; ${ }^{3}$ Biostatistics Department-Delegations for Clinical Research \\ and Innovation, Lille Catholic Hospitals, Lille; ${ }^{4}$ Pediatric Orthopaedics Department, Children's Hospital Purpan, Toulouse; and \\ ${ }^{5}$ Orthopedic Department, Cannes Hospital, Cannes, France
}

\begin{abstract}
OBJECTIVE The best predictors of height gain due to surgical correction are the number of fused vertebrae and the degrees of the corrected Cobb angle. Existing studies of predictive models measured the radiographic spinal height and did not report the clinical height gain. The aims of this study were to determine the best predictive factors of clinical height gain before surgical correction, construct a predictive model using patient population data for machine learning, and test the performance of this model on a validation population.
\end{abstract}

METHODS The authors reviewed 145 medical records of consecutive patients who underwent surgery that included placement of posterior spinal instrumentation and fusion for idiopathic scoliosis between 2012 and 2016. Standing and sitting clinical heights were measured before and after surgery in patients who had been surgically treated under similar conditions. Multivariate analysis was then performed and the results were used to develop a predictive model for height gain after surgery. The data from the included patients were randomly assigned to a learning set or a test set.

RESULTS In total, 116 patients were included in the analysis, for whom the average postoperative clinical height gain in a standing position was $4.2 \pm 1.8 \mathrm{~cm}$ (range $0-11 \mathrm{~cm}$ ). The best prediction model was calculated as follows: standing clinical height gain $(\mathrm{cm})=1-0.023 \times$ sitting clinical height $(\mathrm{cm})-0.19 \times$ Risser stage $+0.058 \times$ Cobb preoperative angle $\left(^{\circ}\right)+0.021 \times \mathrm{T} 5-12$ kyphosis $\left({ }^{\circ}\right)+0.14 \times$ number of levels fused. In the validation cohort, $91 \%$ of the predicted values had an error of less than one-half of the actual height gain.

CONCLUSIONS This predictive model formula for calculating the potential postoperative height gain after surgical treatment can be used preoperatively to inform idiopathic scoliosis patients of what outcomes they may expect from posterior spinal instrumentation and fusion (taking into account the model's uncertainty).

https://thejns.org/doi/abs/10.3171/2020.3.SPINE191541

KEYWORDS prognosis study; deformity; spine; scoliosis; idiopathic; pediatrics; adolescent; height; posterior instrumentation; correction fusion

$\mathrm{H}$ EIGHT plays a major role in social integration. ${ }^{1}$ Most adolescents worry about their cosmetic appearance, ${ }^{2}$ and potential height gain following surgical correction of scoliosis is a frequent concern of adolescent idiopathic scoliosis patients and of their parents. Several studies have quantified the loss of height induced by the progression of spinal curvature ${ }^{3-5}$ or the potential height gain due to surgical correction of low thoracic kyphosis. ${ }^{5}$ However, prediction of postoperative height gain is still controversial. According to our search of the literature, 5 prediction models have been reported. ${ }^{6-10}$ Thus far, the best predictors of height gain resulting from surgical correction were the number of fused vertebrae $e^{7-10}$ and the de- grees of the corrected Cobb angle ${ }^{6,9}$ or postoperative Cobb angle. ${ }^{7,8}$ Nevertheless, the corrected Cobb angle can only be measured after the surgery, when height gain prediction is less relevant, especially if the surgeon wants to provide an estimation of height gain to those patients considering surgery. Furthermore, the authors of those predictive models measured the radiographic spinal height and did not report the clinical height gain, which could be of greater interest to the patient. Clinical height measurement is a reliable and rapid technique, ${ }^{11,12}$ which is easily accessible in consultation, contrary to measurement on radiographs, which require controlling for magnification error. ${ }^{9}$ The aims of this study were to determine the best predictive 
factors of clinical height gain before surgical correction, construct a predictive model using machine learning with actual patient population data, and test the performance of this model on a validation population.

\section{Methods}

\section{Study Design and Setting}

This study was approved by the internal review board of Lille Catholic Hospitals. A retrospective prognostic study was performed between January 2012 and April 2016. All medical records of idiopathic scoliosis patients who were treated for surgical correction at our institute by one surgeon (C.M.) were reviewed. Clinical height data were collected within 15 days before surgery, as well as immediately after and 3 months later. Radiographic studies were performed 5 days before and 3 months after surgery.

\section{Study Patients}

In the reviewed medical records, we identified 145 consecutive patients with idiopathic adolescent scoliosis who underwent surgery during the inclusion period. All patients underwent posterior spinal fusion. Patients were excluded if any of the following data were not available in the database: preoperative and postoperative frontal and lateral full-body radiographs, preoperative elongation radiographs, and preoperative and postoperative clinical height measurements. Elongation radiographs were performed in the supine position with bipolar elongation, with a loading of $10 \mathrm{~kg}$. Patients who underwent preoperative halo elongation were excluded. All parents signed an informed consent to data processing authorization.

\section{Surgical Technique}

All patients underwent a posterior approach surgery that included placement of posterior spinal instrumentation and fusion for idiopathic scoliosis. Fusion was performed using mixed instrumentation with both pedicle screw and hook constructs. ${ }^{13}$

\section{Outcome Measures}

Standing (Fig. 1A) and sitting (Fig. 1B) clinical heights were determined before surgery and within 15 days and at 3 months postoperatively. To limit measurement bias, clinical height was measured by the same operator under similar conditions: the patient was barefoot in the Frankfort horizontal plane, ${ }^{11,14}$ and the same stadiometer fixed to the wall was used for all patients.

Moreover, to corroborate the validity of this clinical measurement, the radiographic height gain was measured for those patients who had frontal and lateral biplanar low-dose radiographs acquired with an EOS system (EOS Imaging). ${ }^{15}$ This technology allows imaging of the patient in a calibrated space that can be used to perform reliable 3D measurements which are not affected by magnification artifacts. The T1-L5 vertical spinal height was measured pre- and postoperatively in 3D from the middle of the superior endplate of $\mathrm{T} 1$ to the middle of the inferior endplate of L5 (Fig. 2). Measurements were performed by

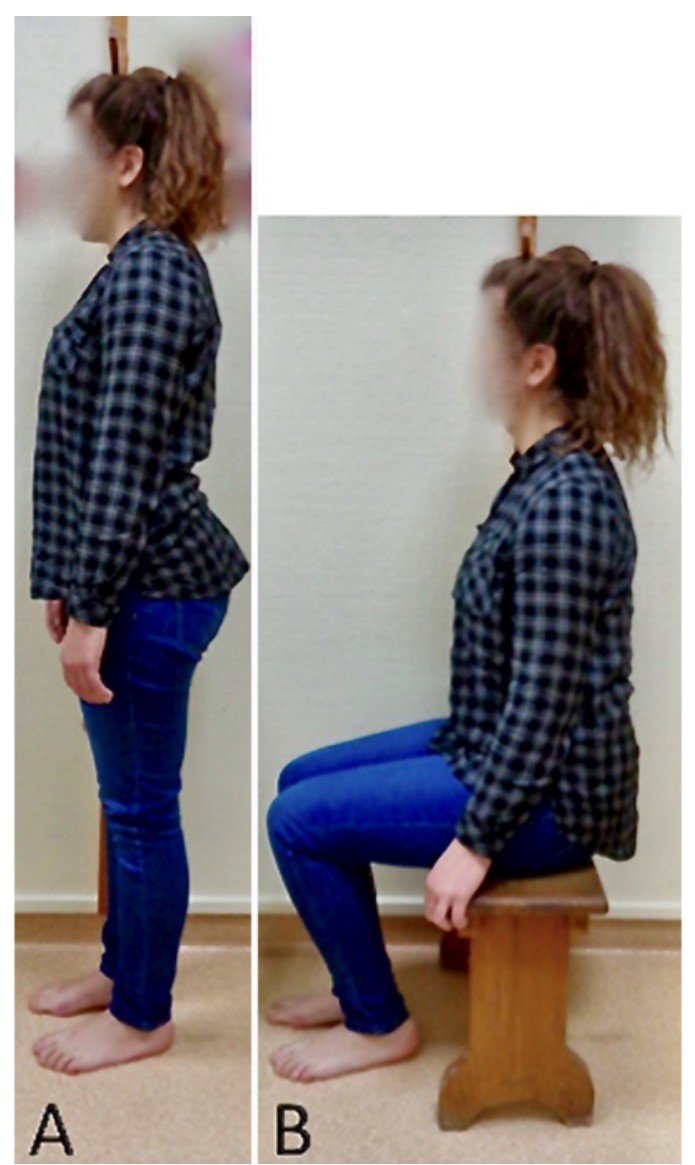

FIG. 1. Measurement of a patient's standing (A) and sitting (B) clinical height with a stadiometer. Figure is available in color online only.

a fully trained operator who was not the patient's surgeon or the operator who measured the patient's clinical height.

For all patients, including those for whom only conventional radiography was available, radiographic measurements were performed with fully integrated, digitally calibrated angular and linear measurement instruments (Synapse; Fujifilm Medical Systems). On the preoperative radiographs, the Cobb angle of the major curves and the T5-12 kyphosis were measured with the standard techniques $^{9}$ (Fig. 3A and B). On elongation radiographs, the Cobb angle was measured on the same limit vertebrae as those appearing on the preoperative radiograph (Fig. 3C). On postoperative radiographs, the number of levels fused and the number of implants (screws and hooks) were recorded. Scoliotic curves were classified according to the Lenke $^{16}$ method. Radiographic data were collected by a single surgeon (T.L.; not the operating surgeon).

\section{Statistical Analysis}

For continuous variables, the mean \pm standard deviation and range were calculated (clinical standing and sitting heights, preoperative and elongation test Cobb angle, and T5-12 kyphosis), and for discrete variables the number and proportion were calculated (sex, Risser sign, numbers of levels fused, and number of implants). 

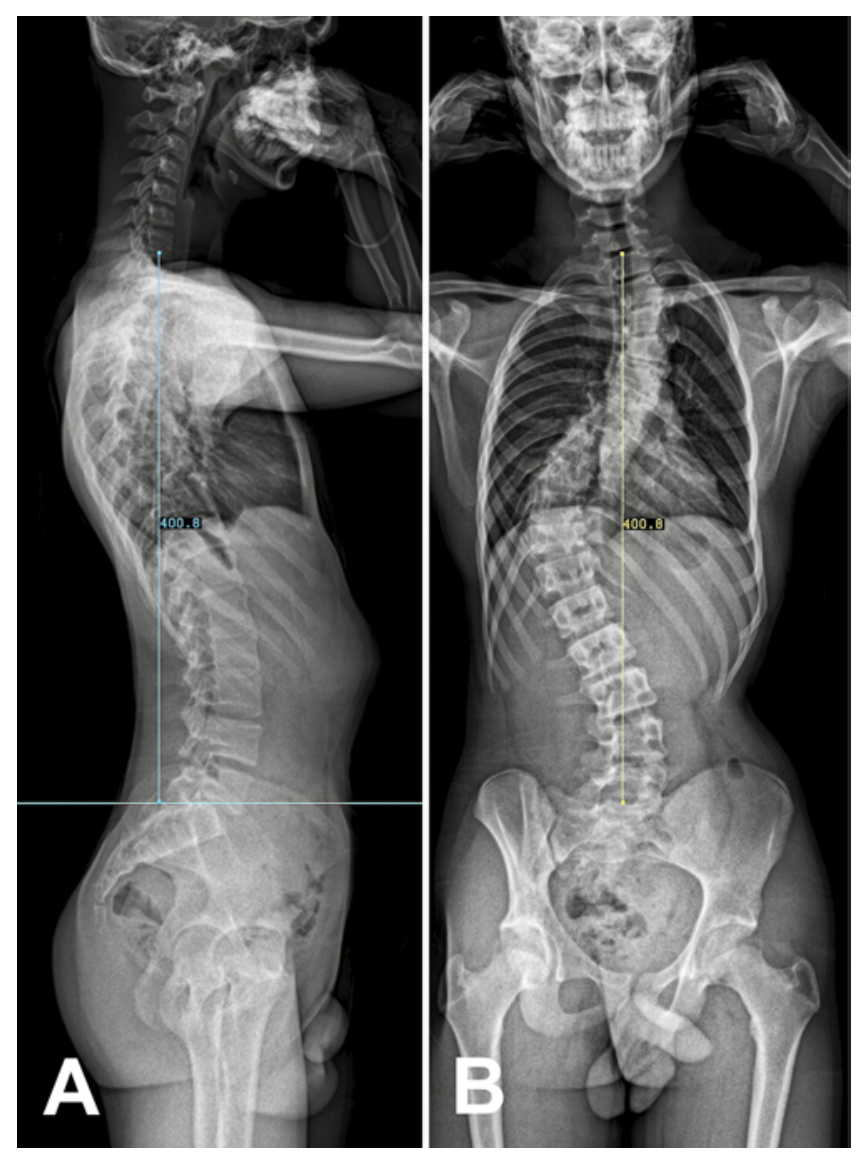

FIG. 2. Preoperative measurement of the T1-L5 distance (vertical line) on a biplanar low-dose radiograph. The measurement is performed simultaneously in the lateral (A) and frontal (B) radiographs to improve accuracy (3D measurement). The horizontal line corresponds to a level marker to facilitate simultaneous measurement on the lateral and frontal radiographs. Figure is available in color online only.

The link between these continuous parameters and the patient height gain was studied. For continuous variables, the nonparametric Spearman rank correlation coefficient was calculated between each variable and patient height gain, and a test of the nullity of the coefficient was performed. To assess the influence of the patient's sex, the height gains for male and female patients were compared with a Wilcoxon Mann-Whitney test, as the data were not normally distributed.

Multivariate analysis was then performed, the aim being to get a predictive model for the height gain after surgery. All the data for each included patient were randomly assigned to a learning set (data for $70 \%$ of the patients were used for model learning) and a test set (data for $30 \%$ of the patients were used to independently assess the model performance).

Data for the various prediction methods were added to the machine learning set, with performances being measured by using the $\mathrm{R}^{2}$ coefficient and estimated by a leaveone-out strategy, to reduce the risk of overestimation due to overfitting. The methods used were linear regression (with feature selection based on Akaike and Bayesian in- formation criteria, and penalized), regression tree, random forest, support vector machines, Bayesian tree, and conditional inference tree. The performance of the model that most closely matched the actual patient outcome data was then measured on the test set.

The comparability of the test and the learning sets was assessed by using a Student t-test or Wilcoxon MannWhitney test (in case of nongaussian data). R software (version 3.2.5; the R Foundation for Statistical Computing) was used.

\section{Results \\ Study Population}

In total, 116 patients were included in the analysis. Their mean age was $15.8 \pm 1.9$ years (range 11.9-21.3 years) at the time of surgery, with 104 female patients (90\%) and 12 male patients $(10 \%)$. All Lenke curve types ${ }^{16}$ were represented (Lenke 1/2 thoracic curves, 67 patients; Lenke 3/4/6 thoracic and lumbar curves, 39 patients; Lenke 5 thoracolumbar or lumbar curves, 10 patients). Twenty-three patients (20\%) had a Risser ${ }^{17}$ stage $\leq 2$ and $93(80 \%)$ had a Risser stage $\geq 3$. The mean preoperative clinical height was $160.9 \pm 7.5 \mathrm{~cm}$ (range 140-179 $\mathrm{cm}$ ) in the standing position and $82.6 \pm 4 \mathrm{~cm}$ (range 73-92.5 cm) in the sitting position. The mean preoperative Cobb angle was $61.6^{\circ} \pm$ $13.6^{\circ}$ (range $31^{\circ}-111^{\circ}$, with only 2 major curves $<40^{\circ}$ ) and the mean preoperative T5-12 kyphosis was $26.8^{\circ} \pm 15.8^{\circ}$ (range $-6^{\circ}$ to $69^{\circ}$ ). The apex of thoracic kyphosis was T8 in $20 \%$ of patients, $\mathrm{T} 7$ in $38 \%$, T6 in $26 \%$, T5 in $14 \%$, and $\mathrm{T} 4$ in $2 \%$ of patients. The average Cobb angle reduction under elongation was $17.6^{\circ} \pm 8.6^{\circ}$ (range $0^{\circ}-38^{\circ}$ ). On average, $12.1 \pm 2.4$ levels were fused (range $7-16$ levels) with $14.9 \pm 2.5$ implants (range 9-21 implants), with a mean number of screws of $7.4 \pm 2.3$ (range $0-12$ screws).

\section{Reliability of Measurement}

Forty measurements of the T1-L5 vertical spinal height were performed pre- and postoperatively in 20 consecutive patients ( 2 measurements per patient, 1 preoperatively and 1 postoperatively). The Pearson correlation was 0.97 (95\% CI 0.90-0.99) between clinical and radiographic height gain. Only 1 patient had a difference in clinical and radiographic height gain greater than $1 \mathrm{~cm}(1.2 \mathrm{~cm})$, while the absolute average difference between the methods was $0.36 \pm 0.3 \mathrm{~cm}$.

\section{Clinical Height Gain and Predictive Factors}

The postoperative average clinical height gain in the standing position was $4.2 \pm 1.8 \mathrm{~cm}$ (range $0-11 \mathrm{~cm}$ ), and in the sitting position it was $3.3 \pm 1.8 \mathrm{~cm}$ (range -1 to $9.5 \mathrm{~cm}$; Fig. 4). According to the Spearman correlation test, the preoperative Cobb angle $(\rho=0.66, p<0.0001)$, number of levels fused $(\rho=0.51, p<0.0001)$, number of implants $(\rho=0.42, p<0.0001)$, and T5-12 kyphosis $(\rho=0.4, p<$ 0.0001 ) were positive predictive factors of clinical postoperative height. There were also negative statistical correlations with sitting preoperative clinical height $(\rho=-0.45$, $\mathrm{p}<0.0001)$, Risser stage $(\rho=-0.40, \mathrm{p}<0.0001)$, age $(\rho$ $=-0.38, \mathrm{p}<0.0001)$, and standing preoperative clinical height $(\rho=-0.22, p<0.0001)$. There was no significant 


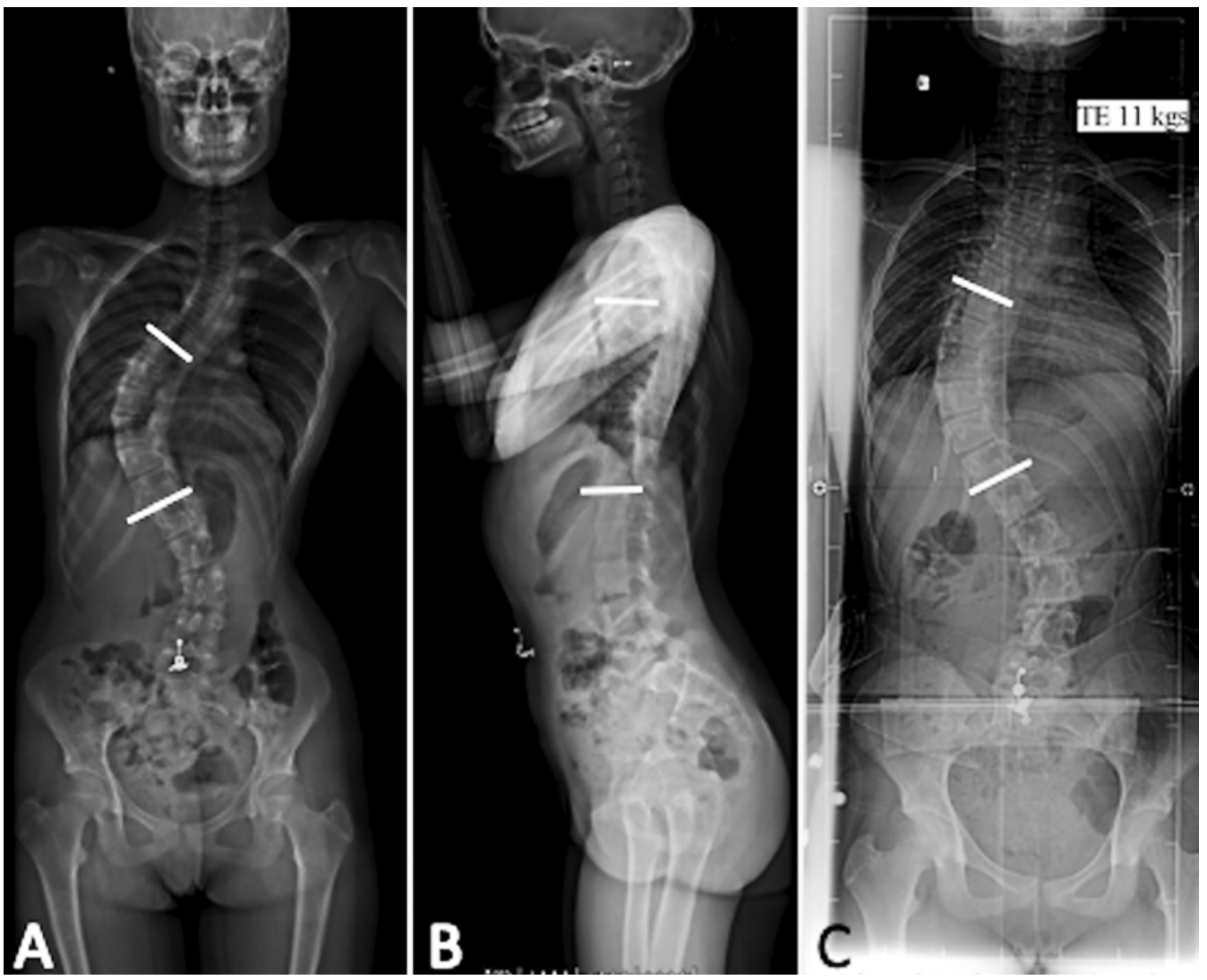

FIG. 3. Examples of preoperative Cobb angle measurements performed with the patient in the standing position (A) and in elongation (C) and measurement of T5-12 kyphosis (B). The white lines indicate limit vertebrae.

statistical correlation with the number of screws $(\rho=0.13$, $\mathrm{p}<0.18)$ and the Cobb angle reduction under elongation $(\rho=0.12, p<0.2)$. No differences in clinical height gain by sex $(\mathrm{p}=0.22)$, type of curve (Lenke $1 / 2,4.1 \pm 1.7 \mathrm{~cm}$; Lenke 3/4/6, $4.3 \pm 2.1 \mathrm{~cm}$; and Lenke 5, $4.1 \pm 0.7 \mathrm{~cm}$; p > $0.2)$, or apex of thoracic kyphosis (T8, $4.0 \pm 1.5 \mathrm{~cm}$; T7, $3.9 \pm 1.2 \mathrm{~cm}$; T6, $4.4 \pm 0.8 \mathrm{~cm}$; T5, $4.7 \pm 1.9 \mathrm{~cm}$; and T4, $4.0 \pm 1.5 \mathrm{~cm} ; \mathrm{p}>0.3)$.

\section{Predictive Model}

The best prediction model was issued from an elastic net penalized regression, with $\mathrm{R}^{2}=0.52$ and a leave-oneout $\mathrm{R}^{2}$ of 0.40 . The model was as follows: standing clinical height gain $(\mathrm{cm})=1-0.023 \times$ sitting clinical height $(\mathrm{cm})-$ $0.19 \times$ Risser stage $+0.058 \times$ Cobb preoperative angle $\left(^{\circ}\right)+$ $0.021 \times$ T5-12 kyphosis $\left(^{\circ}\right)+0.14 \times$ number of levels fused.

\section{Test Performance of Model}

On the validation cohort, $91 \%$ of the predicted values had an error less than one-half of the actual height gain, and for $50 \%$ of the values the error was less than $20 \%$ (Fig. 5). The $\mathrm{R}^{2}$ computed on the validation set was 0.47 (error range formula $[-1.9 ; 2.5]$, mean absolute error 0.93 , root mean square error 1.1). Moreover, no relationship was observed between the percentage of Cobb angle correction and the model error (correlation coefficient -0.04).

\section{Discussion}

In this original study, the predictive factors correlated with postsurgery clinical height gain in patients with idiopathic adolescent scoliosis were determined. A multilinear model for predicting clinical height gain was developed and its reliability was determined; its use in the clinical routine is straightforward since it is a linear combination of clinical (sitting height) and radiological measurements (Risser sign, Cobb preoperative angle, kyphosis, and number of levels fused). We insist on the fact that the clinical height gain does not motivate the surgery but makes it possible to answer an important question frequently asked by patients and their families.

Preoperative Cobb angle, number of levels fused, and preoperative T5-12 kyphosis were correlated with clinical height gain. These correlations have already been described in the literature. ${ }^{6-10}$ However, the correlation between kyphosis and lordosis with height gain remains controversial in numerous studies., ${ }^{9,10}$ This is not surprising, because idiopathic thoracic scoliosis is responsible for thoracic lordosis in most cases.

The numbers of implants were also correlated to clinical height gain. To our knowledge, this factor has never been described previously. ${ }^{6-9}$ The surgical technique employed consisted of hybrid constructs with implants set up only at strategic locations previously defined by Cotrel 

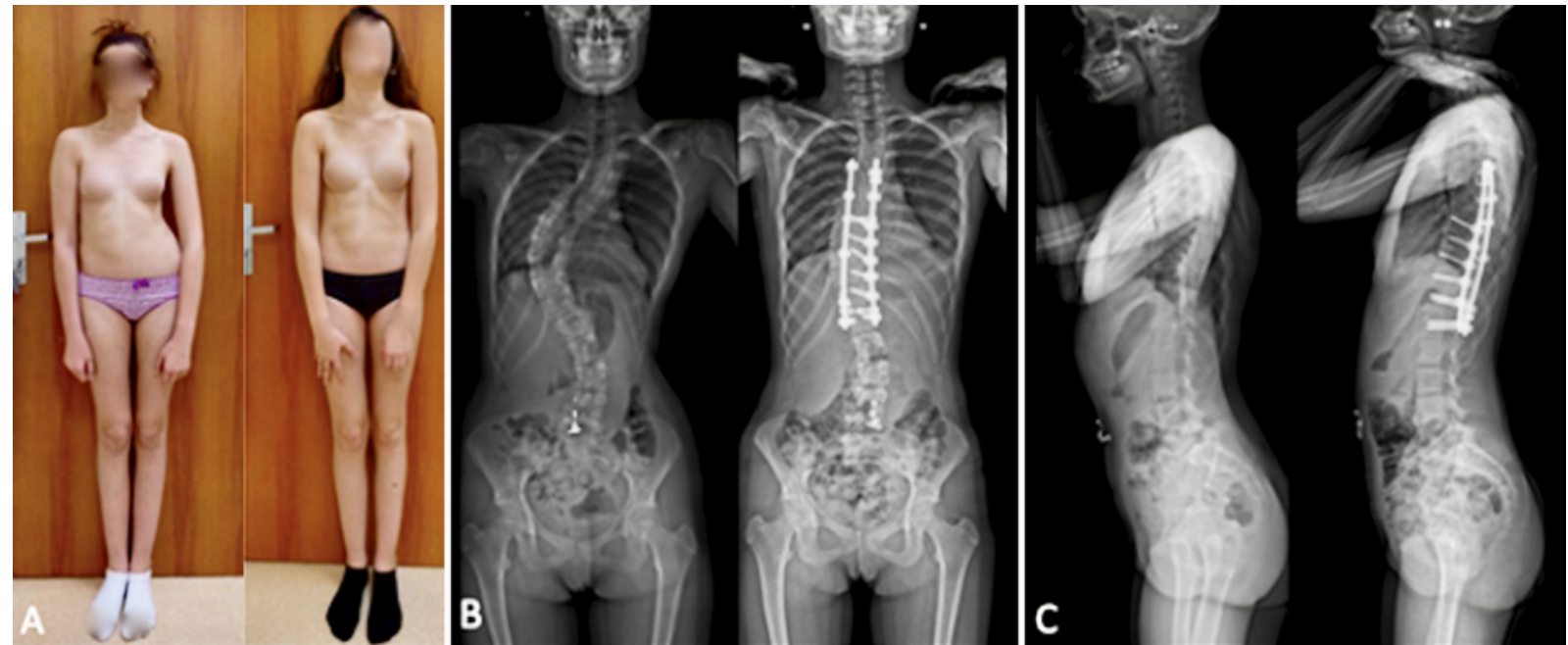

FIG. 4. Example of clinical height gain after a correction with fusion of T5-L1 (A), with frontal (B) and sagittal (C) pre- and postoperative radiographs. Figure is available in color online only.

and Dubousset. ${ }^{13}$ The present values of clinical height gain (average $4.2 \mathrm{~cm}$ ) can be compared with the values resulting from a construct with implants on all levels. Spencer et al. ${ }^{9}$ used screws at all levels except proximally, where hooks were used, obtaining an average height gain of 2.71 $\mathrm{cm}$. Van Popta et al..$^{10}$ found a mean gain of $4.6 \mathrm{~cm}$, but no information was given on the type of constructs used. Watanabe et al. ${ }^{6}$ found an average height gain of $3.24 \mathrm{~cm}$ after hybrid instrumentation.

Although we found that the Risser stage was negatively correlated with height gain, no correlation was observed between the reducibility of the curvature of the elongation test and height gain. First, this could be due to the fact that in adolescent idiopathic scoliosis the spine is relatively flexible and therefore the elongation factor did not have a significant effect on height gain. Second, younger patients with a lower Risser stage gained more height after surgery because they had more severe deformities at the time of surgery (Cobb angle $70^{\circ} \pm 13^{\circ}$ in Risser $0-3$ vs $57^{\circ} \pm 11^{\circ}$ in Risser 4-5; the difference was not statistically significant). Therefore, earlier surgery was indicated for these patients.

The predictive model of clinical standing height gain (cm) after posterior correction fusion only depends on preoperative factors, unlike previous models found in the literature which were based on the percentage of frontal ${ }^{6,9}$ or sagittal correction obtained, ${ }^{8}$ or on the presence of osteotomies. ${ }^{8}$ The model reported by Spencer et al. ${ }^{9}$ focuses on the height gain with good reliability $\left(\mathrm{R}^{2}=0.85\right)$ but includes the degree of correction of the frontal Cobb angle, which is a measure taken from postoperative data. The model reported by Watanabe et al. ${ }^{6}$ is also based on analysis of the corrected Cobb angle. The present model was built to inform the patient quickly and easily in preoperative consultation; it showed a lower adjusted $\mathrm{R}^{2}(0.47)$, but we can predict the height gain. In the van Popta et al. ${ }^{10}$ study, the authors aimed to predict postoperative height
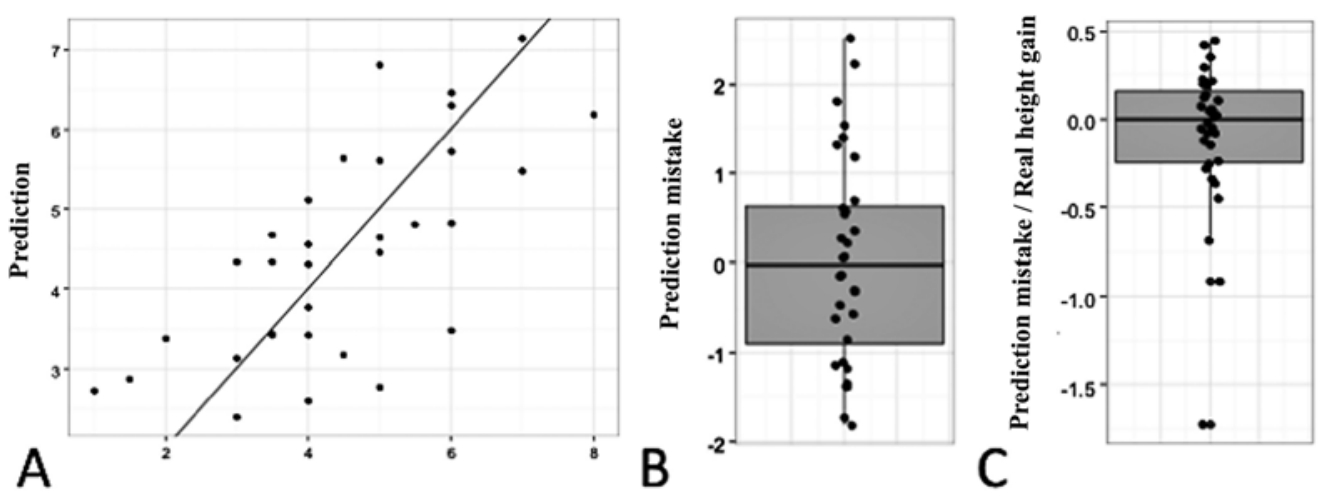

FIG. 5. A: Prediction based on the observed clinical height gain for the validation population. The $x$-axis corresponds to the actual height gain in centimeters and the y-axis corresponds to the predicted height gain in centimeters. B and C: Box plots representing the prediction mistake (error; B) and the ratio between the prediction error and the actual height gain for the validation population (C). Grey boxes correspond to IQRs, box boundaries to $25 \%$ and $75 \%$ quantiles, and black horizontal lines to medians. Vertical lines show ranges from minimum to maximum values, not including outliers. 
but not height gain. This difference makes it possible to obtain a high adjusted $\mathrm{R}^{2}(0.76)$ because the postoperative height is strongly correlated with the preoperative height.

The present work is original because the model was built on a learning population and tested on a validation population. In order to inform patients of the possible error in the preoperative estimate of the postoperative height gain, a margin of error was established for this prediction model. This margin of error reflects the difficulty of finding a reliable and accurate model due to the relatively high variability of the different variables at play.

On the one hand, clinical and radiological measurements are affected by nonnegligible uncertainties. On the other hand, height gain depends on the surgical approach, which is never $100 \%$ reproducible.

To our knowledge, this is the first reported study that uses clinical height measured with a fixed stadiometer as a criterion for evaluation rather than measurements performed on radiographic images. The measurement method used in the present study reflects the daily clinical reality and the patient's everyday life. To evaluate the reliability of this measurement, biplanar radiographs were used to overcome the effects due to enlargement and the distance of the patient from the transmitter. In the EOS booth (EOS Imaging), the patient's position can be reliably estimated and accounted for, and the vertical T1-L5 distance can be measured accurately between vertebral endplates.

\section{Study Limitations}

This study has some limitations. Patient inclusion was retrospective, but inclusions were consecutive and homogeneous. Radiographic measurements were performed only once by a trained operator, who was blinded to the clinical measurements; although measurement reliability was not established, such radiographic measurements are well known. Another limitation is that no assessments of quality of life and self-esteem were performed. Finally, results should be generalized with care because all surgeries in the presented series were performed by the same orthopedic surgeons. Different surgical approaches might result in higher or lower average height gains.

\section{Conclusions}

In conclusion, this study showed that more frontal and kyphotic deformities affect height gain after a posterior fusion correction of idiopathic scoliosis, and that younger patients (smaller Risser sign) can expect higher clinical height gain. The preoperative predictive model of postoperative clinical height gain was $1-0.023 \times$ sitting clinical height $(\mathrm{cm})-0.19 \times$ Risser stage $+0.058 \times$ Cobb preoperative angle $\left({ }^{\circ}\right)+0.021 \times \mathrm{T} 5-12$ kyphosis $\left({ }^{\circ}\right)+0.14 \times$ numbers of levels fused. The model can be calculated for any patient preoperatively to inform them of their potential height gain (taking into consideration the model's uncertainty). Further work should aim at validating this formula for use with different surgical approaches.

\section{References}

1. Tyrrell J, Jones SE, Beaumont R, et al. Height, body mass index, and socioeconomic status: mendelian randomisation study in UK Biobank. BMJ. 2016;352:1582.

2. Weinstein SL, Dolan LA, Spratt KF, et al. Health and function of patients with untreated idiopathic scoliosis: a 50-year natural history study. JAMA. 2003;289(5):559-567.

3. Bjure J, Nachemson A. Non-treated scoliosis. Clin Orthop Relat Res. 1973;(93):44-52.

4. Stokes IAF. Stature and growth compensation for spinal curvature. Stud Health Technol Inform. 2008;140:48-51.

5. Ylikoski M. Height of girls with adolescent idiopathic scoliosis. Eur Spine J. 2003;12(3):288-291.

6. Watanabe K, Hosogane N, Kawakami N, et al. Increase in spinal longitudinal length by correction surgery for adolescent idiopathic scoliosis. Eur Spine J. 2012;21(10):1920-1925.

7. Sarlak AY, Atmaca H, Musaoğlu R, Veliev EV. The height gain in scoliotic deformity correction: assessed by new predictive formula. Comput Math Methods Med. 2012;2012:167021.

8. Hwang SW, Samdani AF, Lonner BS, et al. A multicenter analysis of factors associated with change in height after adolescent idiopathic scoliosis deformity surgery in 447 patients. J Neurosurg Spine. 2013;18(3):298-302.

9. Spencer HT, Gold ME, Karlin LI, et al. Gain in spinal height from surgical correction of idiopathic scoliosis. J Bone Joint Surg Am. 2014;96(1):59-65.

10. van Popta D, Stephenson J, Verma R. Change in spinal height following correction of adolescent idiopathic scoliosis. Spine J. 2016;16(2):199-203.

11. De Miguel-Etayo P, Mesana MI, Cardon G, et al. Reliability of anthropometric measurements in European preschool children: the ToyBox-study. Obes Rev. 2014;15(suppl 3):67-73.

12. Rodacki CL, Fowler NE, Rodacki AL, Birch K. Technical note: repeatability of measurement in determining stature in sitting and standing postures. Ergonomics. 2001;44(12):1076-1085.

13. Cotrel Y, Dubousset J. A new technic for segmental spinal osteosynthesis using the posterior approach. Article in French. Rev Chir Orthop Repar Appar Mot. 1984;70(6):489-494.

14. Mathur R, Bhat M, Satish V, Parvez M. Cleidocranial dysplasia: case report of three siblings. Int J Clin Pediatr Dent. 2009;2(2):32-39.

15. Dubousset J, Charpak G, Dorion I, et al. A new 2D and 3D imaging approach to musculoskeletal physiology and pathology with low-dose radiation and the standing position: the EOS system. Article in French. Bull Acad Natl Med. 2005;189(2):287-300.

16. Lenke LG, Betz RR, Harms J, et al. Adolescent idiopathic scoliosis: a new classification to determine extent of spinal arthrodesis. J Bone Joint Surg Am. 2001;83(8):1169-1181.

17. Risser JC. The iliac apophysis; an invaluable sign in the management of scoliosis. Clin Orthop. 1958;11(11):111-119.

\section{Disclosures}

The authors report no conflict of interest concerning the materials or methods used in this study or the findings specified in this paper.

\section{Author Contributions}

Conception and design: Morin. Analysis and interpretation of data: Compagnon. Drafting the article: Langlais. Critically revising the article: Ursu, Vergari. Reviewed submitted version of manuscript: Ursu, Vergari, Barret. Statistical analysis: Verdun. Study supervision: Morin. English editing and review: Barret.

\section{Correspondence}

Tristan Langlais: Institut Calot, Fondation Hopale, Berck-sur-Mer, France.tristanlanglais@yahoo.fr. 